\title{
HgtSIM: A simulator for horizontal gene transfer (HGT) in microbial communities
}

\author{
Weizhi Song ${ }^{1,2}$, Kerrin Steensen ${ }^{1,3}$, Torsten Thomas ${ }^{\text {Corresp. } 1,4}$ \\ ${ }^{1}$ Centre for Marine Bio-Innovation, University of New South Wales, Sydney, Australia \\ 2 School of Biotechnology and Biomolecular Sciences, University of New South Wales, Sydney, Australia \\ 3 Department of Genomic and Applied Microbiology, Georg-August Universität Göttingen, Göttingen, Germany \\ 4 School of Biological, Earth and Environmental Sciences, University of New South Wales, Sydney, Australia \\ Corresponding Author: Torsten Thomas \\ Email address: t.thomas@unsw.edu.au
}

The development and application of metagenomic approaches have provided an opportunity to study and define horizontal gene transfer (HGT) on the level of microbial communities. However, no current metagenomic data simulation tools offers the option to introduce defined HGT within a microbial community. Here, we present HgtSIM, a pipeline to simulate HGT event among microbial community members with user-defined mutation levels. It was developed for testing and benchmarking pipelines for recovering HGTs from complex microbial datasets. HgtSIM is implemented in Python3 and is freely available at: https://github.com/songweizhi/HgtSIM . 
1 HgtSIM: A simulator for horizontal gene transfer (HGT) in microbial communities

2

3 Weizhi Song ${ }^{1,2}$, Kerrin Steensen ${ }^{1,3}$ and Torsten Thomas ${ }^{1,4}$

$4{ }^{1}$ Centre for Marine Bio-Innovation, ${ }^{2}$ School of Biotechnology and Biomolecular Sciences and

$5{ }^{4}$ School of Biological, Earth and Environmental Sciences, University of New South Wales,

6 Sydney, Australia. ${ }^{3}$ Department of Genomic and Applied Microbiology, Georg-August-University

7 Göttingen, Göttingen, Germany.

8 Corresponding Author: Torsten Thomas (t.thomas@unsw.edu.au )

9

10

11

12

13

14

15

16

17

18

19

20

21

22

23 


\section{Abstract}

27 The development and application of metagenomic approaches have provided an opportunity to 28 study and define horizontal gene transfer (HGT) on the level of microbial communities. However, 29 no current metagenomic data simulation tools offer the option to introduce defined HGT within a 30 microbial community. Here, we present HgtSIM, a pipeline to simulate HGT events among 31 microbial community members with user-defined mutation levels. It was developed for testing and 32 benchmarking pipelines for recovering HGTs from complex microbial datasets. HgtSIM is 33 implemented in Python3 and is freely available at: https://github.com/songweizhi/HgtSIM.

\section{Introduction}

36 Horizontal gene transfer (HGT) has been recognized as an important force in microbial evolution 37 and adaptation (Soucy et al., 2015). A number of pipelines have been developed to identify HGTs 38 in draft or completed genomes of isolated microorganisms (Adato et al., 2015; Hasan et al., 2012; 39 Podell and Gaasterland, 2007; Ravenhall et al., 2015; Trappe et al., 2016; Zhu et al., 2014). In 40 recent years, the development and application of metagenomic approaches have provided novel 41 and vast amounts of information on the genomic composition of uncultured microorganisms 42 (Thomas et al., 2012). This offers an opportunity to study HGT on the level of microbial 43 communities, however new bioinformatics tools and pipelines have to be developed to reliably 44 detect any HGT events in metagenomic datasets. Simulations of metagenomics reads have been 45 essential for the development and benchmarking of pipelines for the quality control, assembly and 46 annotation of metagenomic data (Peng et al. 2012; Kang et al. 2015). These simulation tools 
47 typically produce reads based on defined sets of reference genomes with user-defined abundance

48 distributions and often considering realistic error models for common sequencing technologies

49 (Escalona et al., 2016). However, no current simulation tool offers the option to introduce defined

50 HGT within the microbial community data simulated, thus allowing to test pipelines that aim to

51 detect HGT. Here, we have developed a pipeline called HgtSIM, which can simulate HGTs

52 between the genomes of microbial communities. The pipeline can simulate HGTs with different

53 degrees of similarity for transferred genes found in donor and recipient genomes, thus allowing to

54 assess the detection of relatively recent or past transfers.

\section{Methods}

\subsection{Simulation of gene mutations}

58 The transfer of genes into a recipient genome often involves subsequent mutations that reflect

59 evolutionary drift or adaptation to the new genomic context (e.g. change in codon usage to match tRNA availability). To simulate such mutations without disrupting reading frames and to confine

61 the mutations to a defined range, we use codons as units of mutations. The mutations of codons

62 were grouped into four categories $\left.\left(C_{\mathrm{i}}\right): 1\right)$ one-base, silent mutation; 2) one-base, non-silent 63 mutation; 3) two-bases mutations and 4) three-bases mutations (Table 1).

Table 1 Mutation types of codons

\begin{tabular}{|c|c|c|c|}
\hline Category & Mutation type & Example & Total number \\
\hline $\mathrm{C}_{1}$ & one-base, silent & ATC $($ Ile $) \rightarrow$ ATA (Ile) & 124 \\
\hline $\mathrm{C}_{2}$ & one-base, non-silent & GCC (Ala) $\rightarrow$ ACC (Thr) & 356 \\
\hline \multirow{2}{*}{$\mathrm{C}_{3}$} & Two bases, silent & AGG $(\operatorname{Arg}) \rightarrow \mathbf{C G T}(\operatorname{Arg})$ & 20 \\
\hline & Two bases, non-silent & CTC $($ Leu $) \rightarrow$ CCT (Pro) & 1394 \\
\hline \multirow{2}{*}{$\mathrm{C}_{4}$} & Three bases, silent & AGT (Ser) $\rightarrow$ TCC (Ser) & 12 \\
\hline & Three bases, non-silent & GTG $($ Val $) \rightarrow$ TAC $($ Tyr $)$ & 1400 \\
\hline
\end{tabular}


65 The changed bases are displayed in bold. The corresponding amino acid change is given in parenthesis. As the

66 number of silent two- and three-bases mutations are low $(\approx 1 \%)$ compared to non-silent mutations, we here

67 combined them into the same categories. The start and stop codons were excluded when calculating the number of

68 mutation types.

69

70 The algorithm for simulating random mutations is as follows:

71 (1) Get the length (L) of each gene to be transferred.

72 (2) Define the number of bases need to be changed $(\mathrm{N})$ based on a user-defined identity value

(I) and L: i.e. $\mathrm{N}=\mathrm{LI} / 100$.

(3) Define the type of mutations based on $\mathrm{N}$ and a user-defined ratio of the four mutation categories. For example, if a ratio of $1: 1: 1: 1$ is specified for $C_{1}: C_{2}: C_{3}: C_{4}$, then, $N=C_{1}+C_{2}$

(4) Randomly select $\mathrm{C}_{1}, \mathrm{C}_{2}, \mathrm{C}_{3}$ and $\mathrm{C}_{4}$ codons and perform the corresponding mutations.

All changed nucleotides are recorded in a mutation report file. A BlastP-based comparison

between the amino acid sequences is also provided.

\subsection{Simulation of gene transfers}

82 The steps to simulate random gene transfers are as follows (Figure 1):

(1) Add flanking sequences (if specified) to the (mutated) genes to be transferred. These flanking regions could, for example, be transposon insertion sequences.

(2) Get the total length or the total number of intergenic regions of the recipient genome $(\mathrm{P})$ and user-defined number of genes $(\mathrm{Q})$ to be transferred.

(3) Randomly select Q numbers between 1 and P and cut the recipient genome at corresponding positions to create sub-sequences. If user wants to insert gene transfers only into intergenic regions, then the recipient genome will be cut in the middle position of the selected intergenic regions. 
91 (4) Randomly assign the (mutated) genes to be transferred to the cut points and concatenate them

92 with the sub-sequences.

93 All the break positions and the (mutated) genes inserted to these positions are recorded in an 94 insertion report file.

95

96

97

98

99

100

101

102

103

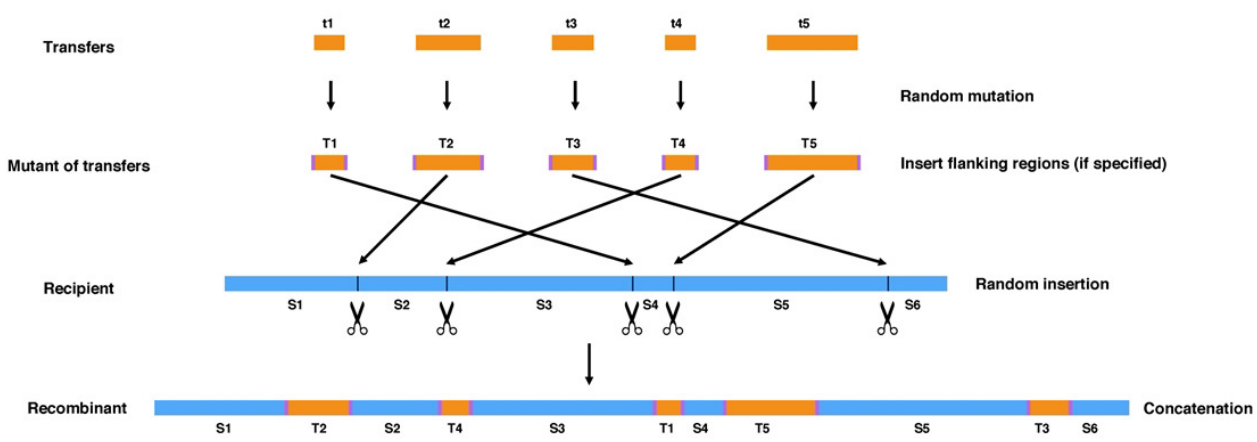

Figure 1 The workflow of HgtSIM.

The Python3 implementation of this HgtSIM algorithm, parameter setting and all scripts used here are available at: https://github.com/songweizhi/HgtSIM.

\section{Results and Discussion}

\subsection{The effect of mutation categories on the level of coded aa changes}

The correlation of mutation on the nucleotide level and the resulting amino acid changes under different ratios of mutation categories were assessed by performing random mutations on 100 genes selected from ten Alphaproteobacteria genomes (Table 2). The values for the level of userdefined nucleotide mutations and the values for the resulting changes in protein sequences were similar for the category ratios of "0:0:0:1" and "1:0:1:1" (Figure 2). This correlation analysis provides the user with information on the level of protein sequence changes that occur at any given nucleotide mutation level and category settings. 


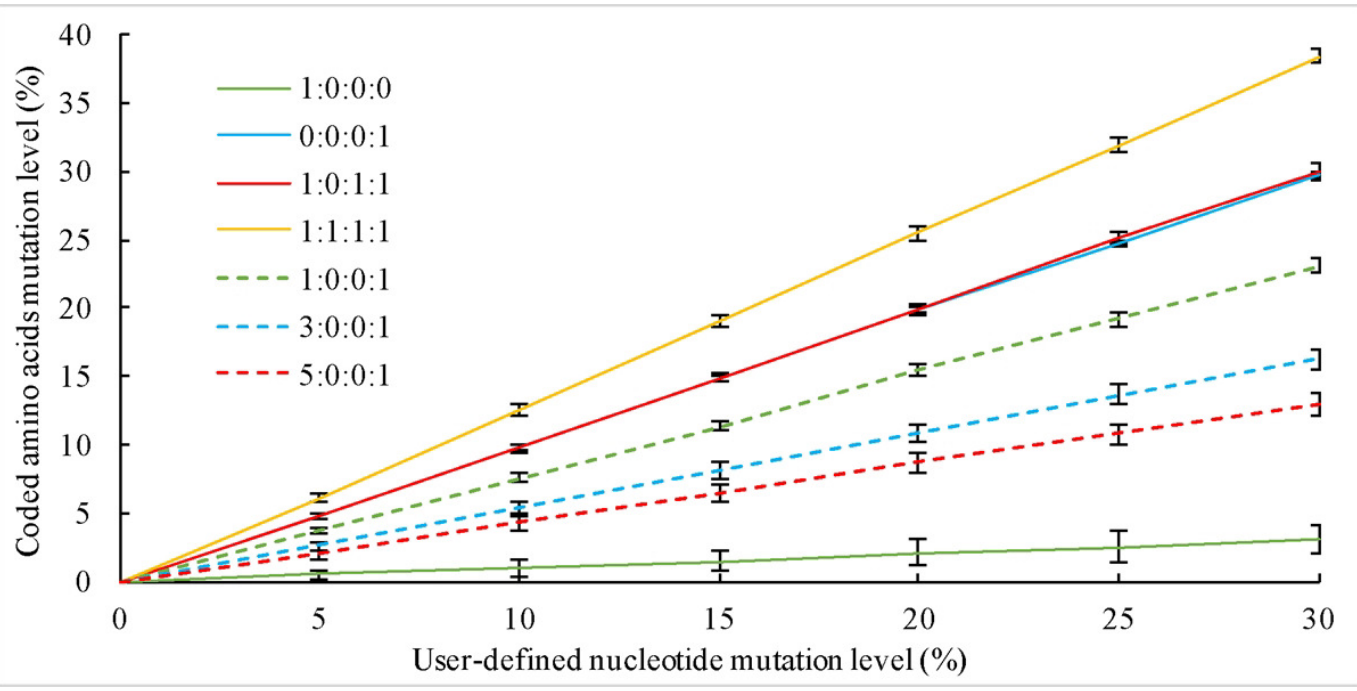

111

112 Figure 2 The correlation of mutation on the nucleotide level and the resulting aa changes under different mutation 113 category ratios. The four numbers separated by colon refer to the ratio between $C_{1}, C_{2}, C_{3}$ and $C_{4}$.

Table 2 The selected 20 genomes

\begin{tabular}{|c|c|c|c|}
\hline Class & Strain & $\begin{array}{c}\text { NCBI } \\
\text { BioProject ID }\end{array}$ & $\begin{array}{c}\text { Genome size } \\
(\mathrm{Mbp})\end{array}$ \\
\hline \multirow{9}{*}{ Alphaproteobacteria } & Acidiphilium multivorum AIU301 & 60101 & 3.58 \\
\hline & Ketogulonigenium vulgarum WSH 001 & 161161 & 2.64 \\
\hline & Mesorhizobium australicum WSM2073 & 47287 & 3.74 \\
\hline & Methylocapsa acidiphila B2 & 72841 & 5.91 \\
\hline & Methyloferula stellata AR4 & 165575 & 4.04 \\
\hline & Rhodovibrio salinarum DSM 9154 & 84315 & 4.30 \\
\hline & Roseobacter litoralis Och 149 & 19357 & 3.98 \\
\hline & Sphingobium japonicum UT26S 1 & 19949 & 3.35 \\
\hline & Starkeya novella DSM 506 & 37659 & 4.54 \\
\hline \multirow{11}{*}{ Betaproteobacteria } & Tistrella mobilis KA081020 065 & 76349 & 3.74 \\
\hline & Alicycliphilus denitrificans $\mathrm{K} 601$ & 50751 & 4.76 \\
\hline & Dechlorosoma suillum PS & 37693 & 3.63 \\
\hline & Gallionella capsiferriformans ES 2 & 32827 & 3.02 \\
\hline & Herbaspirillum seropedicae SmR1 & 47945 & 5.26 \\
\hline & Nitrosospira multiformis ATCC 25196 & 13912 & 3.04 \\
\hline & Ramlibacter tataouinensis ТТВ310 & 16294 & 3.88 \\
\hline & Sideroxydans lithotrophicus ES 1 & 33161 & 2.41 \\
\hline & Snodgrassella alvi wkB2 & 167602 & 2.99 \\
\hline & Sulfuricella denitrificans skB26 & 170011 & 2.86 \\
\hline & Tetrathiobacter kashmirensis WT001 & 67337 & 4.16 \\
\hline
\end{tabular}




\section{3.2 The effect of assemble k-mer range on the recovery of simulated HGTs}

118 We next demonstrated the usefulness of HgtSIM to assess the recovery rate of HGTs from a

119 simulated metagenomic shotgun-sequencing dataset after various sequence assembly processes.

120 For this, 10 genes each were selected from the ten Alphaproteobacteria genomes and randomly

121 transferred to ten Betaproteobacteria genomes (Table 2) with various degrees of mutation $(0 \%$,

$1225 \%, 10 \%, 15 \%, 20 \%, 25 \%$ and $30 \%$ ). The ratio of mutation types was set to $1: 0: 1: 1$ and a flanking

123 sequence of "TAGATGAGTGATTAGTTAGTTA" were added to the two ends of all transfers.

124 Ten million paired-end 100-bp reads (no sequencing error was introduced, corresponding to a

125 coverage of $26.4 \mathrm{x}$ ) with 250 bp insert size were simulated with an in-house script from the 20

126 genomes for each mutation group. To get an even sequencing depth distribution of the 20 genomes,

127 their relative abundances were all set to one. The simulated reads were then assembled with

128 IDBA_UD 1.1.1 (Peng et al. 2012) and metaSPAdes 3.9.0 (Nurk et al. 2017) with multiple k-mer

129 ranges (Figure 3). A gene transfer was considered to be recovered during the assembly if at least

130 one of the gene's two flanking regions was $>1 \mathrm{Kbp}$ and the flanking region matched its recipient

131 genome. To do this, a blastn (Altschul et al. 1990) was performed between the introduced gene

132 transfers and the contigs produced by the assemblers. The blast results were then filtered with an

133 identity cutoff of $99 \%$ and a coverage cutoff of $99 \%$ for the transferred genes.

134 The results show that the best recovery was obtained with a k-mer range of 20-124 bp for

135 IDBA_UD as well as 21-55 and 21-125 bp for metaSPAdes (Figure 3). The number of genes

136 recovered by the two assemblers were decreased when the user-defined nucleotide mutation levels

137 were low (i.e. $<5 \%$ ). When no mutation was introduced, only one and nine genes were recovered 138 by IDBA_UD and metaSPAdes, respectively. 


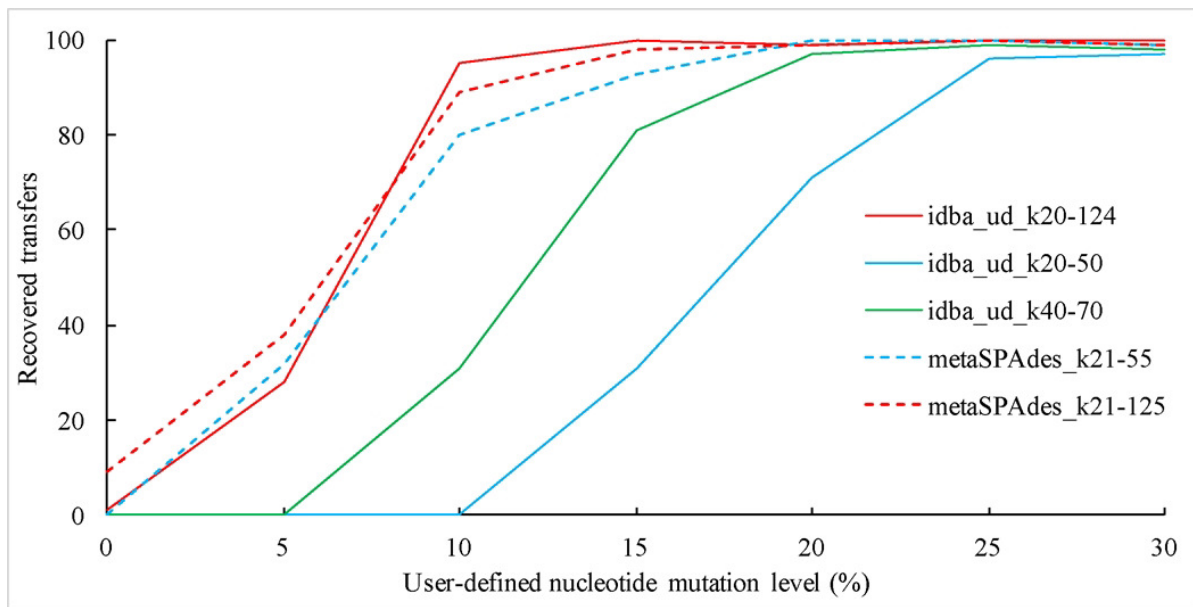

Figure 3 The effect of assemble k-mer range on the recovery of HGT events.

141

\section{3.3 The effect of sequencing depth on the recovery of no-mutation HGTs}

143 We then investigated how sequencing depth might affect the recovery of HGTs with no mutations.

144 To do this, between 1 to 20 million paired-end 100-bp reads with $250 \mathrm{bp}$ insert size were simulated 145 for the 20 genomes, no error was introduced to the simulated reads during simulation and no 146 mutation was introduced to the 100 transferred genes. The simulated reads were then assembled 147 with IDBA_UD and metaSPAdes with the optimal k-mer ranges identified above. Assembly 148 statistics (total length, number of contigs, N50 and percentage of recovered reference genomes) 149 were obtained with MetaQUAST 4.5 (Mikheenko et al. 2015).

151 The results show that the quality of assemblies improved with increased sequencing depth (Figure

$1524 \mathrm{~A}, \mathrm{~B}$ and $\mathrm{C}$ ) and over $98.5 \%$ of sequences for the reference genomes were reconstructed with 153 sequencing depths of greater 6.6x (Figure 4A). We found that the number of gene transfers 154 recovered by IDBA_UD and metaSPAdes was not linearly correlated with sequencing depth. The 155 best recovery (69 out of 100 transfers) was observed with metaSPAdes at a k-mer range of 21-125 156 and sequencing depth of 7.9x (Figure 4D). With a k-mer range of 21-55, 43 gene transfers were 157 recovered by metaSPAdes when the sequencing depth is 4x. As for IDBA_UD, the best recovery 158 was obtained with a sequencing depth of 5.28x. The decrease of HGT recovery rates beyond a 159 certain coverage threshold was surprising and contrasted the improved general quality 160 measurements of the assemblies (Figure 4 A, B and C). One possible explanation is that assemblers 
161 under certain coverage condition are more likely to assemble contigs for the transferred genes that 162 lack flanking regions, and visual inspection of assembly graphs found instances of this.

163

164
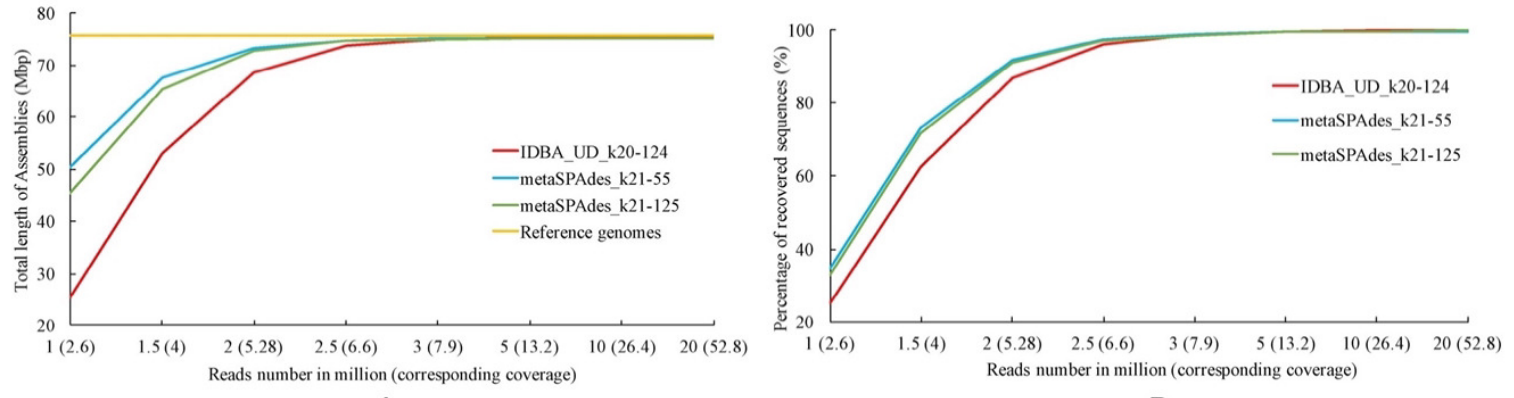

A
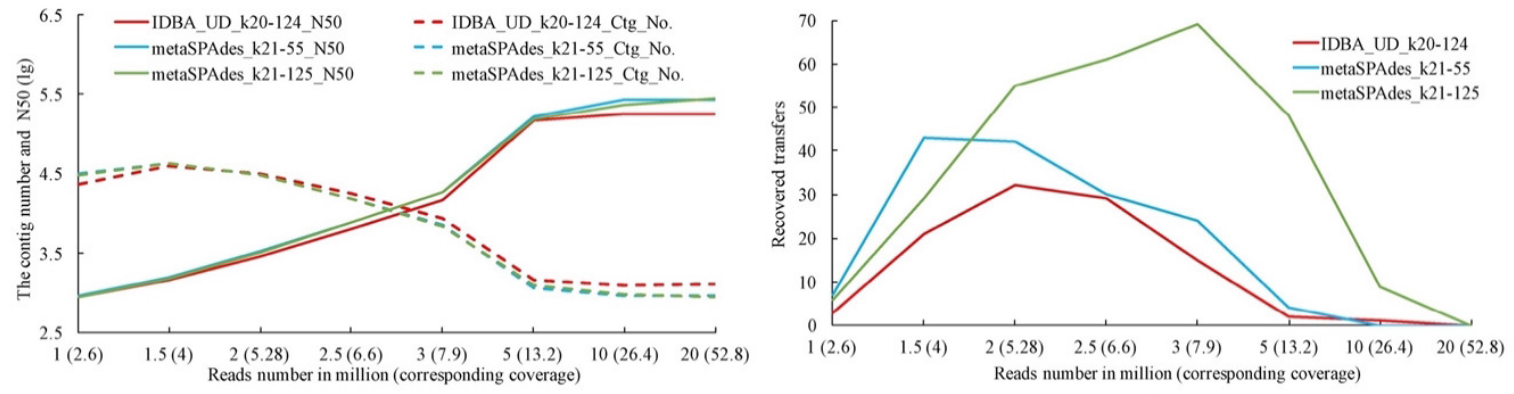

165 C

D

Figure 4 The total length (A), percentage of recovered sequences (B), contig number and N50 (C) of assembler produced assemblies. (D) The number of recovered transfers. The lines show the number of contigs and N50 of metaSPAdes produced assemblies with two different k-mer settings were almost overlapped in panel $\mathbf{C}$.

169

\subsection{The effect of read length and insert size on the recovery of no-mutation HGTs}

171 We also simulated how insert size and read length might influence recovery of transfer events. As

172 the best recovery of no-mutation HGTs was observed with metaSPAdes and k-mer range of 21-

$173125 \mathrm{bp}$ at sequencing depth of 7.9x (Figure 4D), we simulated reads with different length (100 bp and $250 \mathrm{bp})$ and insert sizes $(250 \mathrm{bp}, 500 \mathrm{bp}$ and $1 \mathrm{Kbp})$ to this depth. More no-mutation gene 
Table 3 The effect of reads length and insert size on the recovery of 100 simulated HGT events

\begin{tabular}{ccccccc}
\hline Reads length (bp) & \multicolumn{3}{c}{100} & & \multicolumn{3}{c}{250} \\
\cline { 2 - 4 } \cline { 5 - 6 } Insert size (bp) & 250 & 500 & 1000 \\
\cline { 2 - 7 } Recovered gene transfers & 69 & 55 & 63
\end{tabular}$\quad$\begin{tabular}{cccc}
250 & 500 & 1000 \\
\cline { 4 - 7 }
\end{tabular}

179

$180 \quad 3.5$ The effect of the DNA length on the rate of transfer recovery

181 The correlation between the length of the DNA transferred and its recovery rate was also analyzed.

182 The three datasets shown in Figure 4D were used for this analysis. There was no statistically

183 supported correlation between the gene length and the recovery rate, indicating that gene length

184 has no impact on recovery rate under the given experimental conditions (Figure 5).

185

186

187

188

189

190

191

192

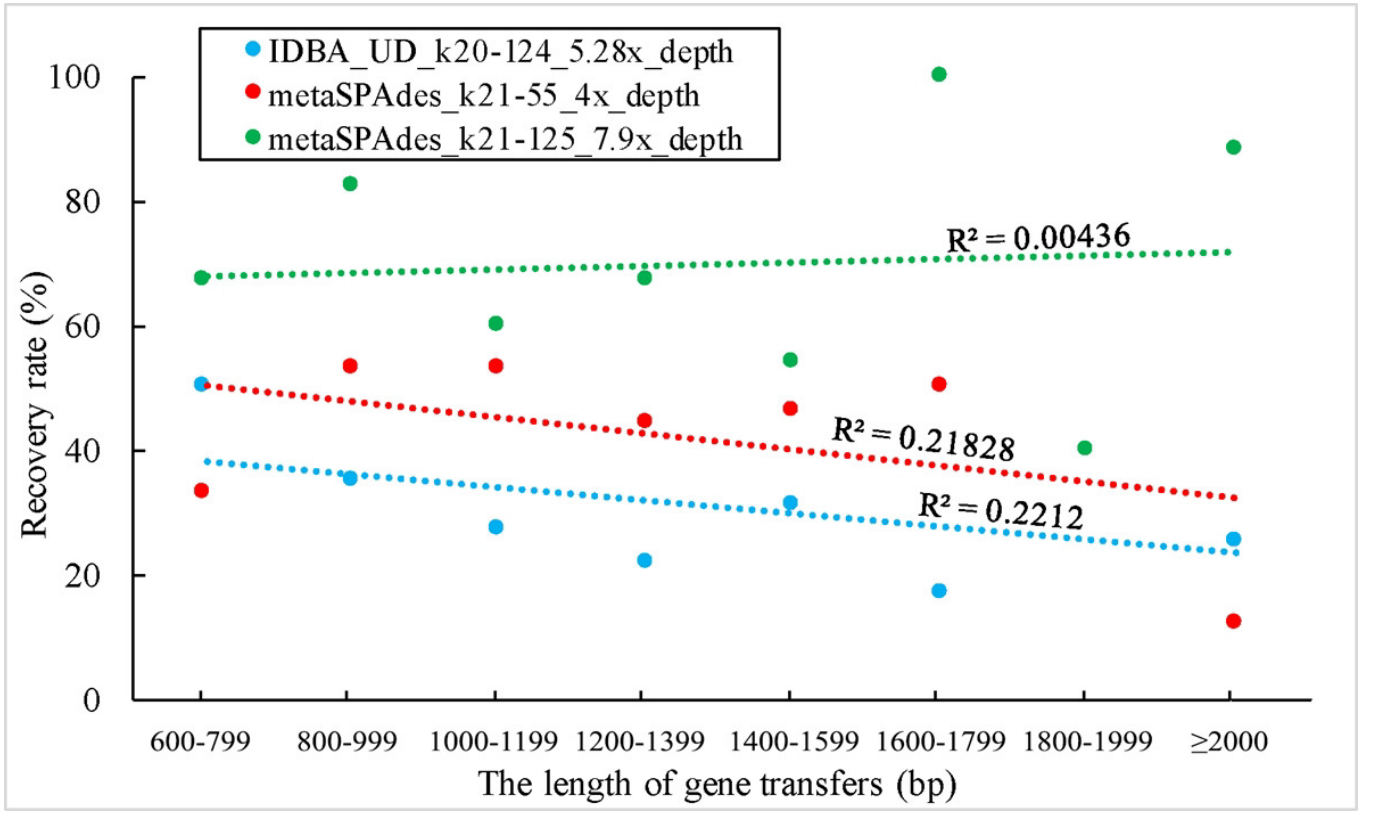

Figure 5 The correlation between the length of gene transfers and their recovery rate

\section{Conclusions}

Our study demonstrates how various aspects of metagenomic sequencing projects (e.g. library production, read length, assembly parameters, gene length) can influence the potential to recover HGT from metagenomic datasets. Testing and benchmarking of various parameters and tools with 
193 simulated datasets produced by HgtSIM will in the future help to develop robust pipelines that

194 have maximal success in recovering HGT from complex metagenomic data.

195

196

References

197

Adato O, Ninyo N, Gophna U, Snir S. 2015. Detecting horizontal gene transfer between closely

198 related taxa. PLoS computational biology 11(10):e1004408.

199

Altschul SF, Gish W, Miller W, Myers EW, Lipman DJ. 1990. Basic local alignment search tool. Journal of molecular biology, 215(3), 403-410.

201

Escalona M, Rocha S, Posada D. 2016. A comparison of tools for the simulation of genomic next-generation sequencing data. Nature Reviews Genetics 17(8):459-69.

Hasan MS, Liu Q, Wang H, Fazekas J, Chen B, Che D. 2012. GIST: Genomic island suite of tools for predicting genomic islands in genomic sequences. Bioinformation 8(4):203.

Kang DD, Froula J, Egan R, Wang Z. 2015. MetaBAT, an efficient tool for accurately reconstructing single genomes from complex microbial communities. PeerJ 3:e1165.

Mikheenko A, Saveliev V, Gurevich A. 2015. MetaQUAST: evaluation of metagenome assemblies. Bioinformatics, 32(7), 1088-1090.

Nurk S, Meleshko D, Korobeynikov A, Pevzner PA. 2017. metaSPAdes: a new versatile 210 metagenomic assembler. Genome Research 27(5):824-34.

211 Peng Y, Leung HC, Yiu SM, Chin FY. 2012. IDBA-UD: a de novo assembler for single-cell and 212 metagenomic sequencing data with highly uneven depth. Bioinformatics 28(11):1420-8.

213 Podell S, Gaasterland T. 2007. DarkHorse: a method for genome-wide prediction of horizontal 214 gene transfer. Genome biology 8(2):R16. 
215 Ravenhall M, Škunca N, Lassalle F, Dessimoz C. 2015. Inferring horizontal gene transfer. PLoS

216 computational biology 11(5):e1004095.

217 Soucy SM, Huang J, Gogarten JP. 2015. Horizontal gene transfer: building the web of life.

218 Nature Reviews Genetics 16(8):472-82.

219 Thomas T, Gilbert J, Meyer F. 2012. Metagenomics-a guide from sampling to data analysis.

220 Microbial informatics and experimentation 2(1):3.

221 Trappe K, Marschall T, Renard BY. 2016. Detecting horizontal gene transfer by mapping

222 sequencing reads across species boundaries. Bioinformatics 32(17):i595-604.

223 Zhu Q, Kosoy M, Dittmar K. 2014. HGTector: an automated method facilitating genome-wide

224 discovery of putative horizontal gene transfers. BMC genomics 15(1):717. 